\title{
Network of social groups or Let's have a party
}

\author{
Marian Brandau and Steffen Trimper \\ Fachbereich Physik, Martin-Luther-Universität,D-06099 Halle Germany.*
}

(Dated: August 8, 2021)

\begin{abstract}
We present a simple model for growing up and depletion of parties due to the permanent communication between the participants of the events. Because of the rapid exchange of information, everybody is able to evaluate its own and and all other parties by means of the list of its friends. Therefore the number of participants at different parties can be changed incessantly. Depending on the deepness of the social contacts, which will be characterized by a parameter $\alpha$, a stable distribution of party members emerges. At a critical $\alpha_{c}$ an abrupt depletion of almost all parties is observed and as the consequence all the peoples are assembled at a single party. The model is based on a hierarchical social network. The probability that a certain person is contacted to another one depends on the social distance introduced within the network and homophily parameter $\alpha$.

PACS numbers: 89.75.Hc; 87.23.Ge; 89.65.-s, 05.65.+b
\end{abstract}

*Electronic address: trimper@physik.uni-halle.de 


\section{INTRODUCTION}

Finding of groups of alike elements in data and in interests is of great importance in all quantitative sciences. Thereby methods and tools of theoretical physics turned out to be successful in giving new insight into the complex behavior in different situations. Models developed in statistical physics proved to be fruitful in reproducing and predicting features of

traffic [1], migration problems [2] as well as in opinion formation within social groups [3], 4]. Although the modeling of opinion formation, based on application of cellular automata, Ising models and other tools of computational physics, is a quite drastic simplification of complicated cognitive processes, the general properties of the system will be reflected [5]. The main goal of the paper dealing with opinion formation, for a review see [5], are interested to figure out a complete consensus or a diversity of final opinions from initially diverging opinion. As a further simplification some authors [3, 6] have used a binary choice for opinions. Thus, in the Sznajd model [6], two people sharing the same opinion tend to convince its (six) neighbors of the pair opinion. A generalization of the model including advertising and aging in a multilayer Sznajd model is discussed in [7]. Another related topic is the ghetto formation studied by means of the two-dimensional Ising model with Kawasaki-exchange dynamics [8]. An extension to multi-cultural societies is discussed recently in 9]. A further enlargement of the model is emerged by allowing many discrete opinions on a network [10, 11]. Hereby the analysis is based on a directed network due to Albert and Barabási 12], for a discussion of networks see also [13].

Our paper is likewise addressed to a relation between networks and dynamics of social groups. In particular, we study different individuals within a social group with conflicting interests. Different to other approaches the conflicts may also occur during the time evolution and not only initially, e.g. physically the interaction is time-dependent. To be specific, let us consider young peoples, planning to participate in a common Saturday party. However they are indecisive at which party they will attend. To make a decision they are forced to establish everlasting contacts to the other members of the society under consideration. Due to the permanent contacts the actual decision of each member is modified permanently. Insofar, we consider a dynamical, time dependent interaction between the members of the group. Obviously, the characterized situation corresponds to the reality where the peoples taking part at a certain party are often bored by the audience of that party. As a consequence 
they decide to leave the present party and to orient towards another one. The change-over to another party is normally triggered by the number of friends attendant at that party. The goal of the present paper is to model such a situation. Specifically, we are interested in depletion of all parties with exception of one party, reported in a German news magazine 14]. Such a kind of phase transition is also observed in a class of models when a strong leader and external impact are present [3]. In our model the phase transition is due to selforganization effects and is not controlled by external environment. Instead of that we have introduced a hierarchical social network discussed recently by Watts et al [15]. This network captures the essential ingredients of a network model of connected population [12, 16, 17, 18]. The construction of such kind of networks is motivated by the observation that individuals within a certain population may be grouped according to their function in the society, for example, their hobby, their occupation, etc. The model offers the phenomena of "six degree of separation" discovered by Travers and Milgram some years ago 19]. Recently the spreading of epidemics within a hierarchical network has been discussed 20]. Here, we analyze a party model on a hierarchical social network under a permanent self-organized interaction between the agents.

\section{THE MODEL}

\section{A. The Society}

Let us characterize the situation we are interested in by a group of predominantly young peoples willing to organize some events like parties. However, they are not up to visit any party. Instead of that their aim is to find out the "best" party at the evening. To decide what the best party is, the peoples communicate permanently with their friends getting stuck at any party. Due to everlasting exchange of opinions by mobile phone everyone is well informed about the status of any party. Each member of the social group standing about such an event is able to evaluate its own party by comparison with other ones. Based on the permanent ability getting the total information, everybody decides on its stay at the present party or to change the party. The decision is strongly influenced by the behavior of subgroups which we identify with the circle of friends. In that sense our model is also a stochastic one because the members of the group make their decision, the disposition to 
change the party stochastically. This kind of emergent behavior is characteristic for a social group and hence, there is an evidence to adopt method of statistical mechanics. Hereby, the ensemble is given by a certain group the members of which are linked by common interests such as to arrange parties. They form a social network within the society. The permanent exchange of information and the subsequent decision to change the party or to stay at the party is related to the interaction between the constituents. Thereby, the distribution of parties with different attractiveness offers an additional interaction for the members of the social group under consideration. To be more specific, let us assume the group consists of $N$ persons where all the friends should be included. Further, the systems contains $V$ nodes and $E \subseteq V \times V$ edges. The network is characterized by the numbers $(V, E)$. The nodes $x^{i} \in V$ represents the agents or the persons of the game, whereas the edges between the nodes stands for the connections or the acquaintances between the persons. Additionally, each node $x^{i}$ is assigned to a set of neighbors $y_{\gamma}^{i}$ with $i \in[1, N]$ and $\gamma=1, \ldots, k$. This set is called the friends. The single adjacency list, denoted by $A_{i} \rightarrow\left(y_{1}^{i}, \ldots, y_{k}^{i}\right)$, consists of all the pairs $\left(x^{i}, y_{1}^{i}\right),\left(x^{i}, y_{2}^{i}\right), \ldots,\left(x^{i}, y_{k}^{i}\right) \in E$ with $i \in[1, N]$. The number $k$ is identified as the degree of the set $x^{i}$. Each adjacency list $A=\left\{A_{i}\right\}$ presents one realization of the network.

\section{B. Hierarchical Networks}

Following the basic idea by Watts et al [15] let us introduce a hierarchical network. The model was motivated by the general structure in the groupings of individuals in a society. Such a classification reflects the deepness of relations for instance by the families, the working team, the hobbies or the home district. The situation is depicted in Fig. 1 schematically [18]. The highest level can be regarded as a population of $N$ individuals or nodes. This $N$ nodes may then be partitioned into $b$ groups, each of them can further divided into $b$ subgroups and so on. After $(\ell-1)$ divisions the structure has a total of $\ell$ levels. The underlying structure ends at a level where an individual belongs to a close functional group of size $g$ where $1 \leq g \leq N$ is typically of the order $10^{1}$ to $10^{2}$. The members belonging to the lowest-level subgroup have the highest change of getting friends or in general becoming similar. As stressed in [20] the division in subgroups is usually not unique. For example, all physicists in a certain university can be classified roughly by their research area but simultaneously they can be grouped geographically based on the region where their institute 
is located. As usually the number of persons taking part in a party (nodes) may thus be characterized by $H$ hierarchies. Each of them takes on the structure shown in Fig. 1, As a further important quantity within a hierarchy let us introduce the social distance $x_{i j}$ that measures the similarity between two nodes $i$ and $j$. In case of different hierarchies we define $x_{i j}=\max _{H} x_{i j}^{H}$. For nodes belonging to the same lowest-level group in a given hierarchy, $x_{i j}=1$, otherwise the social distance is even the number of levels from the lowest for which the nodes belong to the same group.

The probability that a person $x^{i}$ is linked to a person $x^{j}$, specifying that $i$ and $j$ are friends, is established with a probability

$$
P\left(x_{i j}\right)=\frac{\exp \left(-\alpha x_{i j}\right)}{\sum_{n=1}^{\ell} \exp (-\alpha n)}
$$

Here the parameter $\alpha$ is a measure of homophily of the system. With other words, the quantity $\alpha$ characterizes the deepness of the contacts between two nodes (persons) within the system. The probability, given in Eq. (11), guarantees that for $\alpha \gg 1$ only links between nodes with small separation, e.g. such belonging to the same subgroup, are probable, whereas for $\alpha=-\ln b$, links between individuals with any social distance are equally probable. In that case a random network results. For intermediate values of $\alpha$, the network shows small-world features.

The above division process is repeated until a mean number of $\langle z\rangle=\langle g\rangle-1$ links are established for each individual in the system. Here $\langle z\rangle$ is the averaged number of friends within the network. Concluding this section we emphasize the model is characterized by the set of parameters $N, H, b, \ell, g$ and $\langle z\rangle$ with the number of nodes $N=\langle g\rangle b^{\ell-1}$. The quantity $\langle g\rangle$ is the average size of the lowest-level subgroups.

\section{PARTY MODELLING AND RESULTS}

\section{A. The network}

A stressed above the social network will be constructed according to [15]. For a population of $N$ individuals the structure is shown in Fig. 1. The links between different nodes (agents, persons) are made by the following steps. Firstly we chose a node $x^{i}$ from the set of all nodes

randomly. Then we take a link of the distance $x_{i j}$ with the probability given by Eq. (10). In a next step we select from all nodes with the given distance $x_{i j}$ from $x^{i}$ a second node 
$x^{j}$. After this a link between the nodes $x^{i}$ and $x^{j}$ is established. Such a link specifies that $i$ and $j$ are friends, e.g. more mathematically, the node $x^{j}$ is added to the adjacency list $A_{i}$ and the node $x^{i}$ is added to $A_{j}$. This procedure is repeated until the persons in the network exhibit a mean number $\langle z\rangle$ of friends. As the result we obtain a set of adjacency lists $A$ which characterizes the network totally. In Fig. 2 we show the frequency distribution of neighbors (or friends or contacts) for different values of the homophily parameter $\alpha$. Let us point out that the distribution is determined by the network essentially. The results have to be incorporated in the further consideration.

To initialize the parties into the model there exits different possibilities. Once one could chose a special distribution of the parties initially, or there is a preselection in such a manner that some places for parties are favored in advance. This situation is not considered here, because we are interested in the self-organization mechanism. While the parties are in progress, the participants want to come to a decision. Instead of that we apply a random distribution of parties.

In the next step we discuss the procedure for making the decision to leave or to stay at a party. For illustration let us assume that a person $i$ is on a certain party denoted as party number 5 . The friend of $i$, labeled by $j$, is on another party, for instance that one with number 2. As a result of the phone call of $i$ with $j$ there appears two possibilities for $j$. Either $j$ remains on its party or $j$ changes from its party 2 to party 5 . As a criteria for the decision person $j$ disposes of information of its own party, denoted by a and of the other party by communication, characterized by, lets say $\mathbf{b}$. Now we define a function $(\mathbf{a}, \mathbf{b})$, specified below, the result of which is the decision "go", in case the party of $i$ is better than the party of its own party ( $j$ 's party) and is "stay" in the opposite case. In our realization person $x^{j}$ knows the number of friends on its party. Thus let us chose $\mathbf{a}=\#\left(A_{j} \cap M_{j}\right)$, where $M_{j}$ is total number of peoples at the party $j$. Likewise the person $x^{j}$ knows the number of friends on $i$ 's party. Consequently we chose $\mathbf{b}=\#\left(A_{j} \cap M_{i}\right)$, where $M_{i}$ is the total number of peoples at the party $i$. A very simple, but realistic rule, is to take the decision $(\mathbf{a}, \mathbf{b}) \rightarrow$ "go", whenever $\mathbf{a}<\mathbf{b}$. In the opposite case $\mathbf{a} \geq \mathbf{b}$ the decision is "stay". In case the number of friends of the other party is larger than on the own party the person decides to change. 


\section{B. Results}

In this part the results of the simulation are presented. In particular, we want to demonstrate the essential influence of the parameter $\alpha$ introduced in Eq. (11). In Fig. 3 the long-time expansion for the size of parties is shown for $\alpha=-\ln 2$. One observes that one party is the winner of the competition. All other parties deplete during the course of the evening. As depicted in Fig. 4 this situation is not preprogrammed. In that figure the short-time expansion of the party-size is shown for the same parameter $\alpha=-\ln 2$ as in Fig. 3. Not that party will be best one at which initially the most persons have been present. Insofar our model seems to be ergodic. However, this point needs further studies. A very typical situation is offered in Fig. 5. Here the homophily parameter is large $\alpha \gg-\ln b$. As stressed in the last section this guarantees that only links between nodes with small separation are probable. The behavior of the system will be dominated by small or isolated subgroups. The same situation is also observed for other intermediate values of $\alpha$. Now let us consider the case that only party "survives" with higher accuracy. Such a situation is already shown in Fig. 3. In Fig. 6] we show the results for the region $-\ln 2 \leq \alpha \leq 1$. For $\alpha=\alpha_{c} \equiv-0.198$ the system undergoes a phase transition from a single party state for $\alpha=-0.2>\alpha_{c}$ to a multi-party state for $\alpha=-0.197<\alpha_{c}$. The sharp increase is not due an external impact but exclusively by the internal, self-organized interaction between the members of the social group. As demonstrated by a slight modification of the parameter $\alpha$ near to $\alpha_{c}$ the result is stable. A similar phenomenon but in another context was observed in [23].

\section{CONCLUSIONS}

In the present paper we have established a simple model to study the behavior of (young) peoples taking part at different parties within a large city. Due to the mobile phones they are able to exchange information permanently. This fact enables the group an everlasting evaluation of the respective party at which they are present. As a simple but realistic measure of the deepness of contacts we have introduce a list of friends labeled to each person. Governed by the aim, to be at the "best" party, the number of friends at a certain party is casting for a decision to leave or to stay at the party. In according to a majority rule any person decides spontaneously to leave its party. An important role for the social 
contacts between the involved persons is played by the homophily parameter $\alpha$ introduced in Eq. (11). Depending on the value of $\alpha$ we observe different scenarios. There is a critical value $\alpha_{c}$ at which the system offers a phase transition from a multi-party behavior to a singleparty state. It would be interesting to study an analytical approach based on a $q$-state Potts-model as suggested in [21] for fuzzy community structures or [22] in case of financial market simulations.

\section{Acknowledgments}

We acknowledge discussions with Gunter Schütz (Fz. Jülich) and collaboration with Dafang Zheng (Hangzhou). The paper had been supported by the DFG under the grant TR $3000 / 3-3$. 
[1] D. Helbing, Rev. Mod. Phys. 73, 1067 (2001).

[2] W. Weidlich, G. Haag, Concepts and Models of Quantitatively Sociology (Springer, Berlin 1983); W. Weidlich, Phys. Rep. 204, 1 (1991)

[3] K. Kacperski and J. A. Holyst Physica A 269, 511 (1999); ibid 287, 631 (2000);

[4] F. Schweitzer and J. A. Holyst, Eur. J. Phys. B 15, 723 (2000).

[5] D. Stauffer How to convince others? in AIP Conference on the Monte Carlo method in the physical sciences edited by J. E. Gubermatis (2003), cond-mat/0307133.

[6] K. Sznajd-Weron and J. Sznajd, Int. J. Mod. Phys. C 11, 1157 (2000).

[7] C. Schulze, Int. J. Mod. Phys. C 14, 95 (2003).

[8] H. Meyer-Ortmanns, Int. J. Mod. Phys. C 14, 311 (2003).

[9] C. Schulze, Int. J. Mod. Phys. C 16, 351 (2005).

[10] D. Stauffer and H. Meyer-Ortmanns, Int. J. Mod. Phys. C 15, 241 (2004).

[11] D. Stauffer, A. O. Sousa, and C. Schulze Discretized opinion dynamics of Deffuant model on scale-free networks, cond-mat/0310243 (2004).

[12] R. Albert and A.-L. Barabási, Rev. Mod. Phys.74, 47 (2002).

[13] S. N. Dorogovtsev and J. F. F. Mendes, Evolution of Networks (Oxford University Press, New York 2003).

[14] Keine Ahnung ... ich ruf dich an, Der Spiegel 12 (2004).

[15] D. J. Watts, P.S. Dodds, and M. E. J. Newman, Science 296, 1302 (2002).

[16] D. J. Watts and S. H. Strogatz, Nature 393440 (1998).

[17] A.-L. Barabási and R. Albert, Science 286, 509 (1999).

[18] S. H. Strogatz, Nature 410, 268 (2001).

[19] J. Travers and S. Milgram, Sociometry 32, 425 (1969).

[20] D. Zheng, P. M. Hui, S. Trimper and B. Zheng, Physica A 352, 659 (2005).

[21] J. Reichardt and S. Bornholdt, Phys. Rev. Lett. 93, 218701 (2004).

[22] T. Takaishi, Simulations of financial makets in a Potts-like model, cond-mat/0503156.

[23] A. Pluchino, V. Latora and A. Rapisarda, Int. J. Mod. Phys. C 16, 515 (2005) 


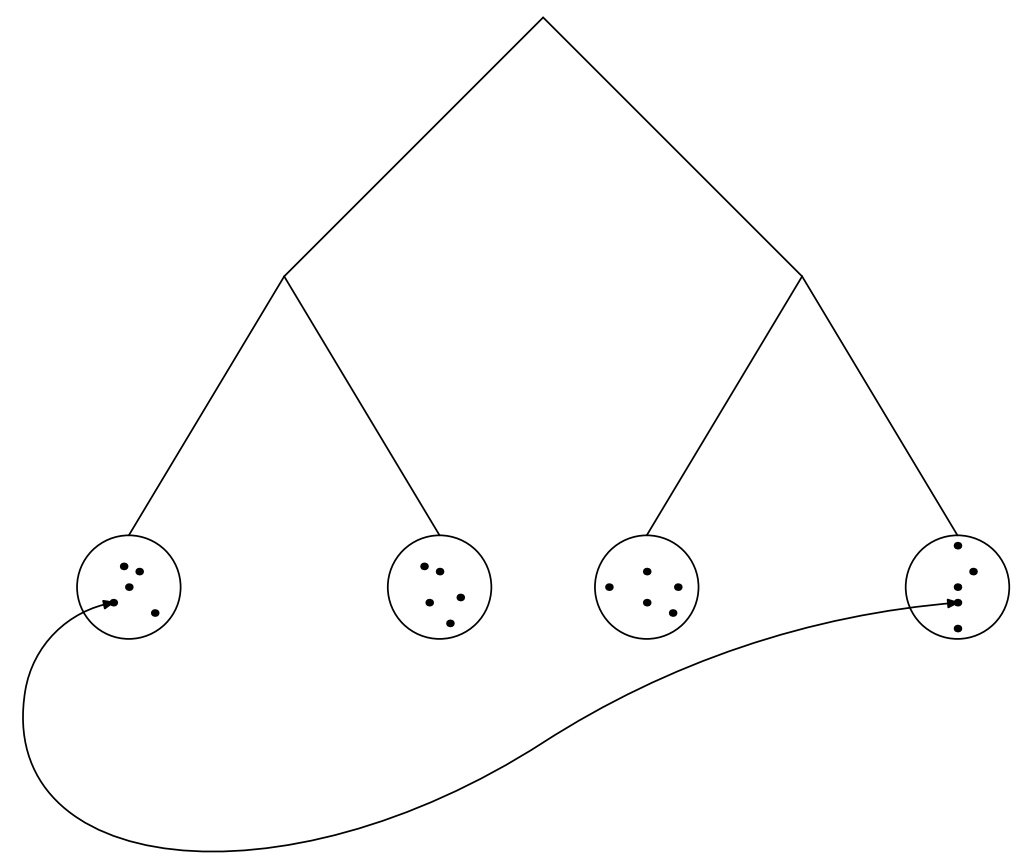

FIG. 1: Schematic diagram of grouping individuals in a hierarchical social network with $\ell=3$. Each group is further divided into $b=2$ subgroups. A group of $N$ nodes are classified into lowestlevel subgroups with $\langle g\rangle=5$. There $x_{i j}$ is the social distance between nodes $i$ and $j$ and is here $x_{i j}=3$. 

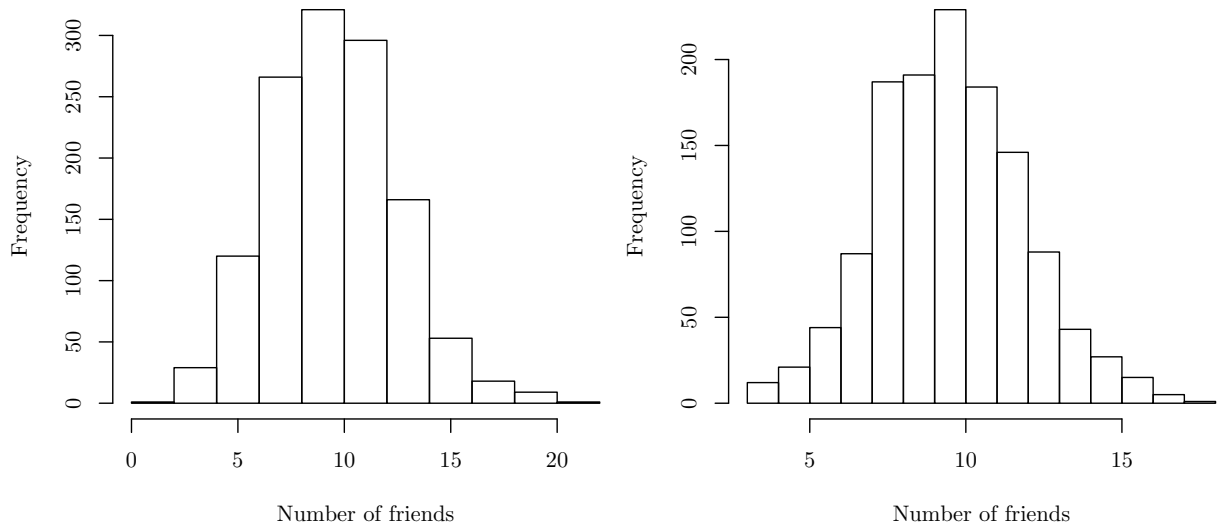

(a)

(b)

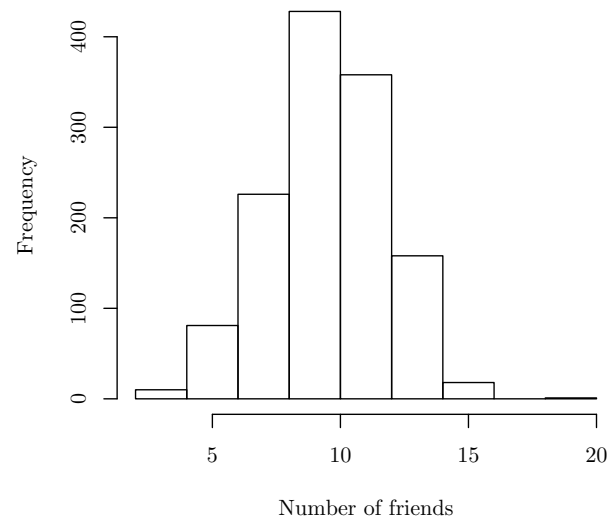

(c)

FIG. 2: Frequency distribution of neighbors or friends for three different parameters $2(\mathrm{a}) \alpha=-\ln 2$, 2(b) $\alpha=1$ and 2(c) $\alpha=10$. The number of nodes is $N=1280(\ell=8)$. The distribution is strongly determined by the network. 




FIG. 3: Long-time evolution of the size of the parties for $\alpha=-\ln b, b=2, H=1$ and $N=5120$. The number of parties is 10 . 


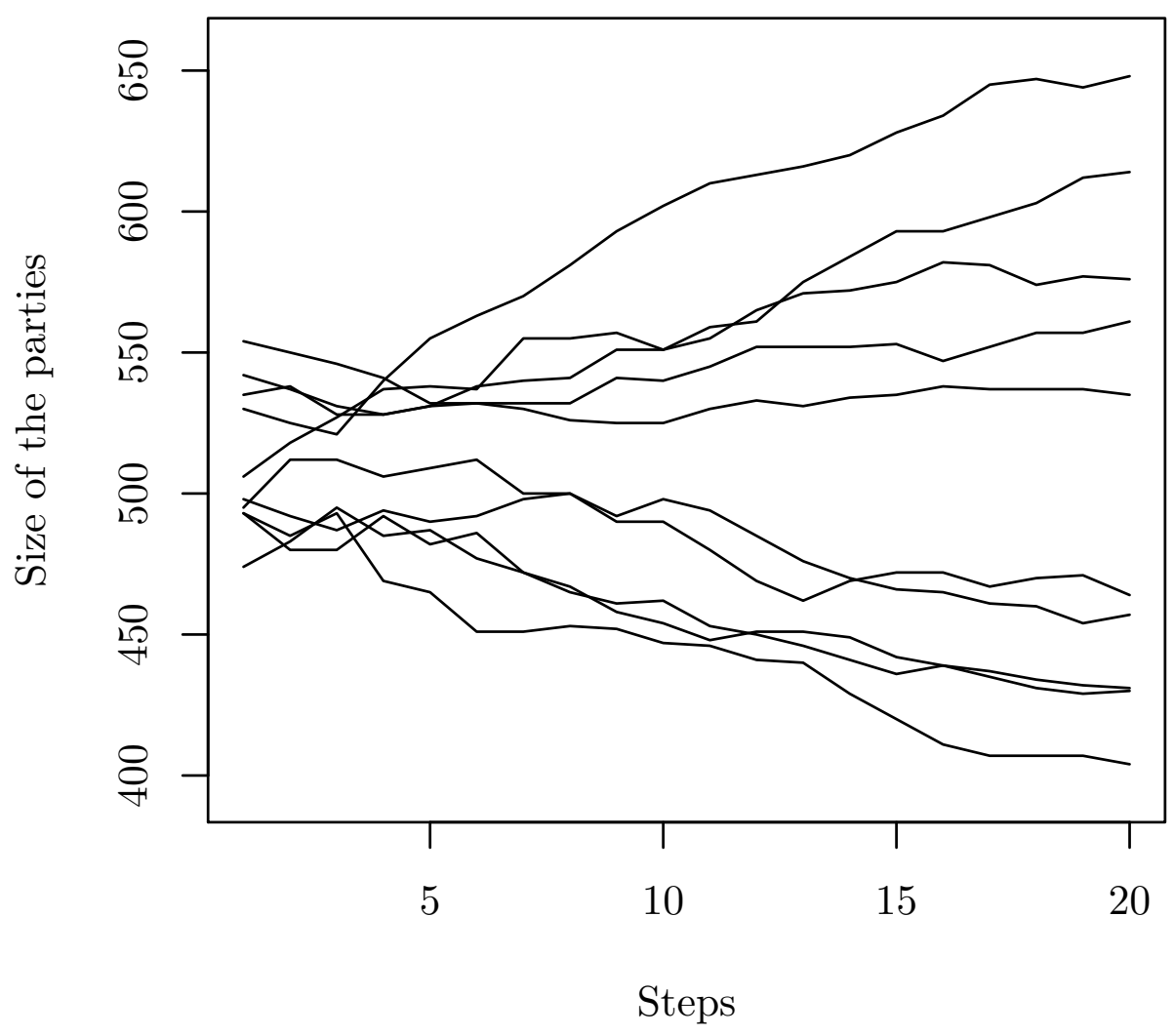

FIG. 4: Short-time expansion of the size of parties for the same parameters as in Fig. 3. Obviously, the biggest party initially will not be the winner in the long-time expansion. 




FIG. 5: Long-time expansion of the size of the parties for large parameter $\alpha=10$. In that case only links between nodes with small separation $x_{i j}$ are probable. Large values for $\alpha$ leads to isolated subgroups of nodes.

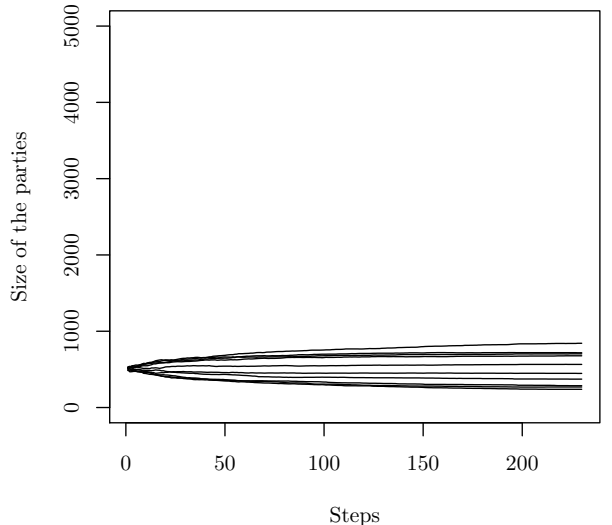

(a)

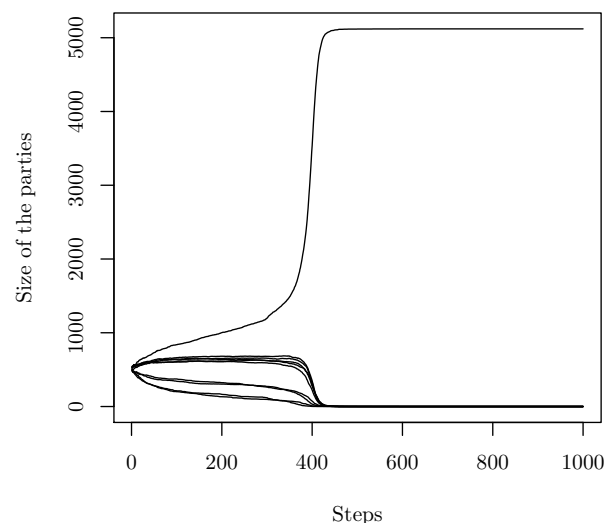

(b)

FIG. 6: Behavior of the size of parties in the vicinity of the critical value $\alpha_{c}=-0.198$. Whereas for 6(b) $\alpha>\alpha_{c}$ only one isolated party survives a many party state is observed for $6(\mathrm{a}) \alpha<\alpha_{c}$. 